\title{
Écouter les femmes enceintes... et après ?
}

\section{Listen to pregnant women....and after?}

\author{
F. Molénat \\ (C) Springer-Verlag France 2012
}

La volonté d'approfondir la communication avec les femmes enceintes répond aux connaissances actuelles sur le devenir de l'enfant : il s'agit d'assurer le bien-être maternel, pour ellemême d'abord, et par là d'améliorer l'environnement humain $\mathrm{du}$ fœtus et du nourrisson. L'impact somatopsychique des échanges précoces entre l'enfant et son environnement est désormais connu ; il rend caduque le débat entre inné et acquis. Donner la parole aux futures mères, prendre en compte ce qu'elles éprouvent, constitue le premier (et souvent suffisant) « soutien à la parentalité » selon la formule consacrée. Recueillir les facteurs de stress, permettre d'en réduire l'intensité tôt dans la grossesse, apporte un gain reconnu en terme de morbidité périnatale. Prévenir la dépression du postpartum relève désormais des objectifs réalistes du suivi global de la naissance.

Le remboursement par l'assurance-maladie d'un temps élargi de dialogue au titre d'une première séance de préparation à la naissance offre une opportunité remarquable. Accueillir les questions des futurs parents, apporter des réponses ajustées, s'informer des points d'appui dont ils disposent, anticiper avec eux leurs besoins spécifiques aux étapes sensibles, activer si nécessaire les intervenants de proximité en vue du retour à domicile... tout ceci relève de l'évidence à portée de main. Que d'économies en perspective si l'on atténue les effets délétères d'une passivité imposée, d'une angoisse non traitée, de la peur de «ne pas y arriver » souvent exprimée. Que de consultations aux urgences évitées en pré ou postpartum - comme en témoignent certaines études ! Que de mieuxêtre chez les professionnels moins isolés... « On écoute mieux lorsqu'on n'est plus seul », témoignent ceux qui ont opéré la bascule. «On est soi-même moins stressé quand la patiente se sent soutenue », dit un médecin. Et les femmes elles-mêmes : " j'ai revu mon médecin. C'était différent. Il m'a écoutée, la sage-femme l'avait appelé $» . .$. On écoute mieux lorsqu'on a les réponses. Un tel état d'esprit relève du bon sens, et pourtant peine à se généraliser.

En effet, dérives, contresens et malentendus entravent grandement l'efficacité de l'entretien prénatal précoce. La

F. Molénat $(\bowtie)$

15, rue Cité-Bousquet, F-34090 Montpellier, France

e-mail : fmolenat@yahoo.fr, www.afree.asso.fr culture du travail en partenariat personnalisé n'y était pas, et l'entretien - porte d'entrée à une coordination qui doit s'organiser lorsqu'elle s'avère nécessaire avec la femme enceinte et son conjoint - est tombé comme un pavé dans la mare. «On ne sait pas par quel bout l'attraper, disait un gynécologue-obstétricien en 2006, mais il nous oblige à réfléchir : comment travailler ensemble ?... ». "On a eu des formations, on nous dit de travailler en réseau, mais comment faire ... ? ». Sur de nombreux terrains, la difficulté reste majeure : la méconnaissance par chacun de la place des autres dans un continuum et l'incapacité fréquente à se relier de manière efficace laissent insatisfait.

De nombreuses enquêtes ont été réalisées par les Réseaux périnatals. Un sérieux contresens apparaît. On s'enquiert du nombre d'orientations vers une assistante sociale ou un psychologue. Une question manque le plus souvent : quel retour au praticien qui suit la grossesse, au médecin traitant qui connaît la famille et assurera la suite ? Certes des supports de transmission s'élaborent ici ou là, mais l'intérêt d'une liaison personnalisée dans les situations de vulnérabilité semble absente en de nombreux sites. Par exemple, avant d'orienter vers un psychologue ou un psychiatre, demande-t-on l'avis du praticien qui suit la grossesse ? Ou du médecin traitant qui connaît bien la famille ? Que cette question évidente n'apparaisse pas en dit long sur le biais introduit : l'entretien ne seraitil conçu que pour orienter? Certes parfois, mais comment et dans quelles conditions ? Dans quelle cohérence d'ensemble? Le premier élément de sécurité, pour la femme, son conjoint, le futur enfant, tient dans cette phrase tant entendue à l'issue d'un suivi coordonné : «on a senti que vous travaillez ensemble, c'était extraordinaire, cela nous a complètement rassurés ». Mots prononcés quand en effet, la sage-femme transmet à l'obstétricien l'élément pertinent, ose appeler le psychiatre qui prescrit, prévient le médecin traitant du suivi proposé et lui demande éventuellement son avis pour une orientation spécialisée,... que le professionnel de PMI transmet ses modalités d'intervention, que le psychologue se rend disponible aux soignants à partir de sa propre analyse d'une problématique délicate. Travailler ensemble, c'est d'abord se parler quand la situation exige de tisser progressivement ce réseau personnalisé articulé sur une meilleure écoute, d'abord avec les 
professionnels de confiance choisis par la famille, auxquels s'ajouteront selon les cas des interventions ciblées sur tel ou tel élément d'insécurité : anesthésiste avec sa technique particulière, mode adapté de préparation à la naissance, pédiatre en anténatal, soutien social... Un coup de fil, un message électronique au bon moment, permettront de huiler les places respectives en veillant à leur lisibilité par le couple. Le temps passé là se retrouvera dans la légèreté des consultations, dans la sécurité éprouvée par les professionnels. Mais anticiper les étapes et activer des fonctions jusqu'alors cloisonnées relève à l'évidence d'un changement de culture.

Il est admis que la transmission de "facteurs de risque " objectifs non assortis des émotions éprouvées dans le présent par les parents, risque de soulever des représentations négatives chez le professionnel qui reçoit l'information sèche, qu'il s'agisse d'un comportement, d'une addiction, d'un diagnostic psychiatrique, d'un traumatisme affectif. Mais, se parler de ce qu'éprouvent les parents, de leurs attentes et de leurs craintes, reste ardu. Pour rester succinct dans une transmission, il faut une idée claire du rôle possible de chaque intervenant et des liens de confiance à améliorer pour chaque cas. Plus on se fait confiance, moins on a besoin de se transmettre d'informations « objectives » relevant de l'intimité ou du comportement. Le nid que constituent les liens interprofessionnels facilitera pour les futurs parents eux-mêmes l'énoncé d'un élément significatif selon la place du consultant. Rappelons l'origine de la démarche : atténuer les effets iatrogènes du cloisonnement, favoriser l'expression d'un mal-être qui ne se disait pas dans les consultations classiques. La peur des parents de déranger, d'être jugé, l'ignorance de l'intérêt porté par le professionnel au registre affectif pourtant fondamental dans l'accueil du nouveau-né, tout ceci freine grandement l'expression de facteurs d'insécurité souvent chargés de culpabilité.

Plusieurs sources d'analyse des pratiques sont disponibles.. D'une part, les enquêtes lancées par les Réseaux régionaux, ainsi que les études et mémoires réalisés par des sagesfemmes ou de jeunes médecins. D'autre part les reprises de cas complexes lors de formation dites « en réseau » réunissant toutes les disciplines autour de l'analyse longitudinale, de la grossesse à la petite enfance, de situations à forte charge émotionnelle ayant engagé de nombreux acteurs. Ces dernières, effectuées devant un public diversifié invité à réagir et à élaborer des hypothèses au fur et à mesure de la présentation prospective (anonyme), constituent une sorte de laboratoire d'étude des pratiques périnatales. Une méthodologie rigoureuse ${ }^{1}$ permet d'identifier les liaisons utiles qui ont pu ou non se réaliser, visant à améliorer la sécurité globale des futurs parents et de l'enfant, mais aussi celle des professionnels. La suite du cas exposé permet de valider les hypothèses de départ. Elles jouent le même rôle que les revues de mortalité-morbidité organisées par les Réseaux périnatals, mais nécessitent une préparation et une animation plus complexes, du fait que se croisent des interventions de disciplines différentes, échelonnées dans le continuum périnatal : médecin généraliste, gynécologue-obstétricien, spécialistes du diagnostic anténatal, échographistes, sagesfemmes de fonction différente, équipes médicosociales, psychologues, psychiatres d'enfant ou d'adulte...

L'objectif à terme est simple : au-delà du respect minimal de la subjectivité des futurs parents, il s'agit de tout mettre en œuvre pour que l'expérience d'un accompagnement attentif et coordonné soit l'occasion d'oser dire leur mal-être sans peur d'une stigmatisation ou d'un jugement, et de faire appel ultérieurement si nécessaire. On sait que le repli sur un malaise à domicile, quelles qu'en soient les causes, risque de se payer très cher sans cette attitude généralisée de prévenance et d'anticipation. Ajoutons que la discipline psychologique - dans la diversité de ses approches - est fortement interpellée pour dégager avec les équipes des points de repère adéquats, et non des concepts issus d'autres champs d'exercice. Travailler avec les autres transforme la pratique et l'enrichit considérablement. Les effets thérapeutiques d'une approche collective coordonnée constituent une nouvelle donne en potentialisant l'efficacité des modèles classiques de soin psychique.

Dernière remarque : on se plaint que les médecins spécialistes ou généralistes ne proposent pas suffisamment la possibilité de rencontrer un professionnel pour un temps de dialogue élargi. Comment peuvent-ils orienter vers un entretien s'ils n'en ont pas éprouvé l'intérêt et l'impact grâce à un retour éclairant sur leur propre compréhension de la situation? L'entretien est un outil pour tous, fait pour renforcer la cohérence du suivi. Quand à la question résurgente du secret, elle ne se pose pas plus ici que dans n'importe quelle consultation médicale, psychologique ou sociale. L'objectif est simple : qu'est-ce qui peut aider le professionnel à garder une vue d'ensemble sur la situation d'une famille en construction, sans avoir à en traiter tous les aspects ? Sûrement pas de dévider une liste de données personnelles, mais plutôt : qui intervient, à quel moment, pourquoi ?

\footnotetext{
${ }^{1}$ Cf Référentiel de formation en réseau (DGS et Société francophone de psychologie périnatale). Sites : www.sfmp.net, www.afree.asso.fr
} 